\title{
Lump Solutions, Multi Lump Solutions and More Soliton Solutions of a Novel (2+1)-dimensional Nonlinear Evolution Equation
}

Hongcai Ma ( $\sim$ hongcaima@hotmail.com )

Donghua University https://orcid.org/0000-0001-8759-5032

Shupan Yue

Donghua University

Yidan Gao

Donghua University

Aiping Deng

Donghua University

\section{Research Article}

Keywords: test functions, lump solutions, multi lump solutions, more soliton solutions, the improved tanh-coth and tan-cot methods

Posted Date: November 30th, 2021

DOl: https://doi.org/10.21203/rs.3.rs-996320/v1

License: (9) This work is licensed under a Creative Commons Attribution 4.0 International License. Read Full License 


\title{
Lump solutions, multi lump solutions and more soliton solutions of a novel $(2+1)$-dimensional nonlinear evolution equation
}

\author{
Hongcai Ma ${ }^{1,2 *}$ Shupan Yue ${ }^{\dagger}$ Yidan Gao and Aiping Deng ${ }^{1,2}$ \\ ${ }^{1}$ Department of Applied Mathematics, Donghua University, Shanghai 201620, China \\ ${ }^{2}$ Institute for Nonlinear Sciences, Donghua University, Shanghai 201620, China
}

\begin{abstract}
Exact solutions of a new (2+1)-dimensional nonlinear evolution equation are studied. Through the Hirota bilinear method, the test function method and the improved tanh-coth and tah-cot method, with the assisstance of symbolic operations, one can obtain the lump solutions, multi lump solutions and more soliton solutions. Finally, by determining different parameters, we draw the three-dimensional plots and density plots at different times.
\end{abstract}

Mathematics Subject Classification. 35G20, 35C05, 35C07.

Keywords: test functions; lump solutions; multi lump solutions; more soliton solutions; the improved tanh-coth and tan-cot methods

\section{Introduction}

Nonlinear evolution equation is considered as a basic model to study many nonlinear phenomena of various complexities, and it is produced in many areas of physics, such as plasma physics, solid state physics and others. From the past to the present, great progress has been made in the research for nonlinear evolution equations, and a great deal of effective methods have been put forward to calculate their exact solutions, for instance, the Hirota's bilinear method [5], the extended homoclinic test function method [22], the inverse scattering and the Darboux transformation [1].

In 1977, lump waves [18] confined to all directions in space have been found for the first time, which has given rise to the attention of numerous scholars. The lump solutions can be obtained by direct bilinear method. Specifically, the auxiliary function for bilinear form is set as a quadratic function. A large number of lump solutions are solved. For example, the specific process of finding lump solutions is shown in the Kadomtsev-Petviashvili (KP) equation [15], the Boussinesq Equation [12], the BKP equation [6], the generalized Bogoyavlensky-Konopelchenko equation [4], the reduced pgbKP and pgKP equations [16] and so on [9-11,13,14]. Compared with soliton solutions, the lump solutions [19,24] are rational function solutions, which decays polynomially in all directions of space, but the lump-type solution $[17,25]$ is localized in mainly all directions of space. In the form of breathing, the multi lump solution $[8,23]$ is a straight

${ }^{*}$ Correspondence should be addressed to Hongcai Ma: Email addresses: hongcaima@hotmail.com

${ }^{\dagger}$ Email addresses: spyue0111@163.com 
line. Using some trigonometric functions and hyperbolic functions, single soliton solutions and multi soliton solutions are obtained by the improved tan-cot and tanh-coth method [3] in the (3+1)-dimensional extended Date- Jimbo-Kashiwara-Miwa(DJKM) equation.

A novel nonlinear evolution equation in (2+1)-dimensional is proposed by [2]

$$
u_{x x x x}+c_{1} u_{t t}+c_{2} u_{x t}+c_{3} u_{x y}+c_{4}\left(u^{2}\right)_{x x}=0 .
$$

where coefficients $c_{i}(1 \leq i \leq 4)$ are arbitrary constants.

If $c_{2}=0, c_{3}=0$, one can get

$$
u_{x x x x}+c_{1} u_{t t}+c_{4}\left(u^{2}\right)_{x x}=0 .
$$

Under the condition $\frac{c_{4}}{c_{1}}=\alpha, c_{1}=\frac{1}{\beta}$, equation (2) becomes Benjamin-Ono equation [21]

$$
u_{t t}+\beta u_{x x x x}+\alpha\left(u^{2}\right)_{x x}=0 .
$$

If $y=x, c_{2}=0$, one can get

$$
u_{x x x x}+c_{1} u_{t t}+c_{3} u_{x x}+c_{4}\left(u^{2}\right)_{x x}=0 .
$$

Under the condition $\frac{c_{4}}{c_{1}}=\alpha, c_{1}=\frac{1}{\beta}, \frac{c_{3}}{c_{1}}=\gamma$, equation (3) becomes the Boussinesq equation [20]

$$
u_{t t}+\alpha\left(u^{2}\right)_{x x}+\beta u_{x x x x}+\gamma u_{x x}=0 .
$$

Taking

$$
u=6(\ln f)_{x x} .
$$

and $c_{4}=1$, the bilinear form for equation (1) is as follows:

$$
\left(D_{x}^{4}+c_{1} D_{t}^{2}+c_{2} D_{x} D_{t}+c_{3} D_{x} D_{y}\right) f \cdot f=0,
$$

where function $f$ is arbitrary with regard to variables $x, y$ and $t$, and the Hirota's bilinear derivative operators $D_{x}, D_{y}$ and $D_{t}$ [7] are constructed by

$$
\begin{aligned}
& D_{x}^{k} D_{y}^{i} D_{t}^{j} f \cdot g=\left(\frac{\partial}{\partial x}-\frac{\partial}{\partial x^{\prime}}\right)^{k}\left(\frac{\partial}{\partial y}-\frac{\partial}{\partial y^{\prime}}\right)^{i}\left(\frac{\partial}{\partial t}-\frac{\partial}{\partial t^{\prime}}\right)^{j} f(x, y, t) \\
& \left.\cdot g\left(x^{\prime}, y^{\prime}, t^{\prime}\right)\right|_{x^{\prime}=x, y^{\prime}=y, t^{\prime}=t .}
\end{aligned}
$$

The m-lump solution is derived from equation (1) by the long wave limit method [2]. This paper intends to research lump solutions, multi lump solutions and more soliton solutions on the basis of bilinear equation(5).

The structure of the paper is divided into five parts. The first section is the background introduction. In the second section, the lump solutions are obtained by quadratic function, and the lump solutions in many cases are discussed. In the third section, the multi lump solution is obtained by exponential function and trigonometric function, and its density diagram is like a pipeline. In Section 4, the more soliton solution is obtained by the improved tanh-coth and tan-cot method. The fifth section is the conclusion. 


\section{Lump solution of equation}

For the purpose of gaining the lump solution for equation (1), we suppose that the quadratic function form of $f(x, y, t)$ is

$$
\begin{aligned}
& f(x, y, t)=\left(a_{1} x+a_{2} y+a_{3} t+a_{4}\right)^{2}+ \\
& \left(a_{5} x+b_{1} y+a_{6} t+b_{2}\right)^{2}+\left(b_{3} x+a_{7} y+a_{8} t+b_{4}\right)^{2}+a_{9} .
\end{aligned}
$$

Substituting the quadratic function form (6) into bilinear equation (5), and making the polynomial coefficients of variables $x, y, t$ are equal to zero. Obviously, we are able to gain a series of algebraic equations about $b_{j}(1 \leq j \leq 4)$ and $a_{i}(1 \leq i \leq 9)$. With the help of mathematical software Maple, by solving the above series of algebraic equations, one can get the following cases:

\section{Case 1.}

$$
\begin{aligned}
& a_{1}=a_{1}, a_{2}=\frac{a_{1} c_{1}\left(a_{6}^{2}-a_{3}^{2}\right)-a_{3} c_{2}\left(a_{1}^{2}+a_{5}^{2}\right)-2 a_{3} a_{5} a_{6} c_{1}}{c_{3}\left(a_{1}^{2}+a_{5}^{2}\right)}, a_{3}=a_{3}, a_{4}=0, \\
& a_{5}=a_{5}, a_{6}=a_{6}, a_{7}=0, a_{8}=0, a_{9}=-\frac{3\left(a_{1}^{6}+3 a_{1}^{4} a_{5}^{2}+3 a_{1}^{2} a_{5}^{4}+a_{5}^{6}\right)}{c_{1}\left(a_{1} a_{6}-a_{3} a_{5}\right)^{2}}, \\
& b_{1}=\frac{a_{5} c_{1}\left(a_{3}^{2}-a_{6}^{2}\right)-a_{6} c_{2}\left(a_{1}^{2}+a_{5}^{2}\right)-2 a_{1} a_{3} a_{6} c_{1}}{c_{3}\left(a_{1}^{2}+a_{5}^{2}\right)}, b_{i}=0(2 \leq i \leq 4) .
\end{aligned}
$$

where $a_{1} a_{5} \neq 0, a_{1} a_{6}-a_{3} a_{5} \neq 0$.

Therefore, by comparing equation (6) and limiting conditions (7), one can write a type of quadratic function solutions of equation (5)

$$
f_{1}=\left(a_{1} x+a_{2} y+a_{3} t\right)^{2}+\left(a_{5} x+b_{1} y+a_{6} t\right)^{2}+a_{9} .
$$

Then, substituting quadratic function (8) into transformation (4), we can obtain

$$
\begin{gathered}
u_{1}=\frac{12\left(a_{1}^{2}+a_{5}^{2}\right)}{\left(a_{1} x+a_{2} y+a_{3} t\right)^{2}+\left(a_{5} x+b_{1} y+a_{6} t\right)^{2}+a_{9}} \\
-\frac{24\left[\left(a_{1} x+a_{2} y+a_{3} t\right) a_{1}+\left(a_{5} x+b_{1} y+a_{6} t\right) a_{5}\right]^{2}}{\left[\left(a_{1} x+a_{2} y+a_{3} t\right)^{2}+\left(a_{5} x+b_{1} y+a_{6} t\right)^{2}+a_{9}\right]^{2}} .
\end{gathered}
$$

Fig. 1 gives three dimensional plots of function (9) and its density plots with a series of parameters $c_{1}=-\frac{15}{32}, c_{2}=\frac{45}{32}, c_{3}=\frac{75}{32}, a_{1}=-\frac{3}{5}, a_{3}=0, a_{5}=\frac{11}{10}, a_{6}=5$ when $t=-1,0,1$.

Fig. 2 gives three dimensional plots of function (9) and its density plots with a series of parameters $c_{1}=-1, c_{2}=2, c_{3}=-\frac{1}{10}, a_{1}=1, a_{3}=-2, a_{5}=3, a_{6}=2$ when $t=-6,0,6$.

\section{Case 2.}

$$
\begin{aligned}
& a_{1}=-\frac{2 a_{3} c_{1}}{c_{2}}, a_{2}=-\frac{a_{3} c_{2}}{c_{3}}, a_{3}=a_{3}, a_{4}=a_{4}, a_{5}=\frac{c_{1}\left(a_{3}^{2}-a_{6}^{2}\right)}{c_{2} a_{6}}, \\
& a_{6}=a_{6}, a_{7}=0, a_{8}=0, a_{9}=-\frac{3\left(a_{3}^{2}+a_{6}^{2}\right)^{4} c_{1}^{3}}{a_{6}^{4} c_{2}^{4} a_{3}^{2}}, b_{i}=0(1 \leq i \leq 4) .
\end{aligned}
$$

where $a_{3} a_{6} \neq 0$.

Therefore, by comparing equation (6) and limiting conditions (10), we can write a type of quadratic function solutions of equation (5)

$$
f_{2}=\left(a_{1} x+a_{2} y+a_{3} t+a_{4}\right)^{2}+\left(a_{5} x+a_{6} t\right)^{2}+a_{9} .
$$




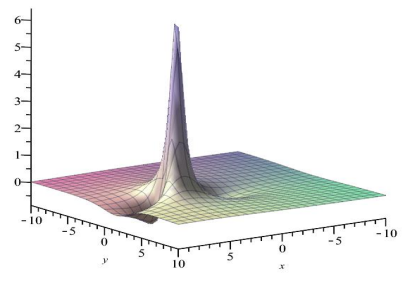

(a)

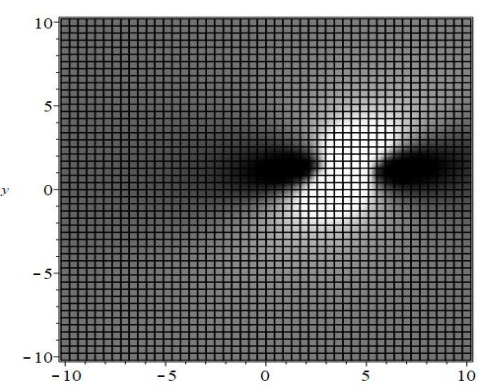

(d)

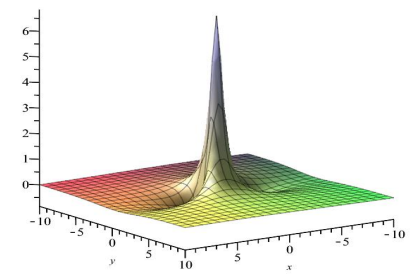

(b)

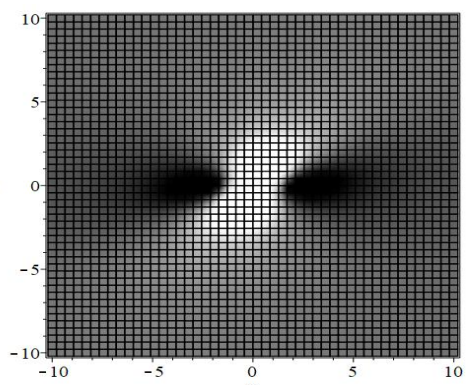

(e)

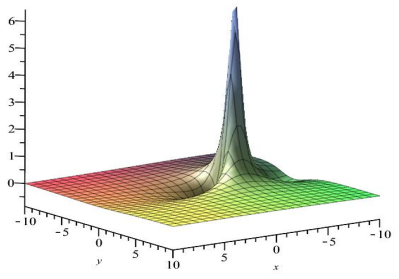

(c)

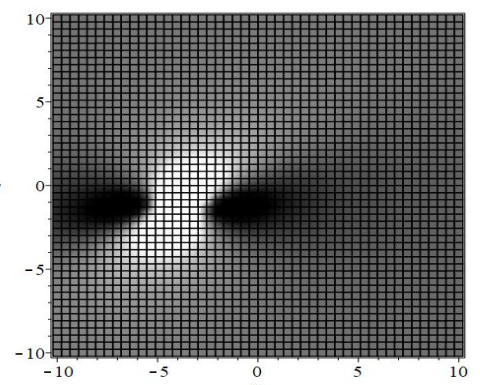

(f)

Figure 1: Three-dimensional plots and density plots of (9) with $c_{1}=-\frac{15}{32}, c_{2}=\frac{45}{32}, c_{3}=\frac{75}{32}, a_{1}=-\frac{3}{5}, a_{3}=0, a_{5}=\frac{11}{10}, a_{6}=5$ when $t=-1$ in (a) and (d), $t=0$ in (b) and (e), $t=1$ in (c) and (f), respectively.

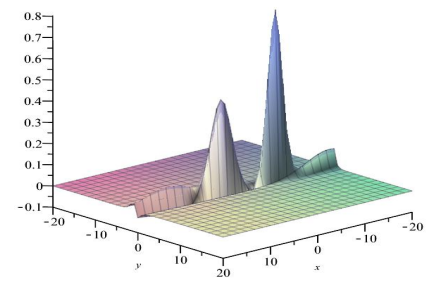

(a)

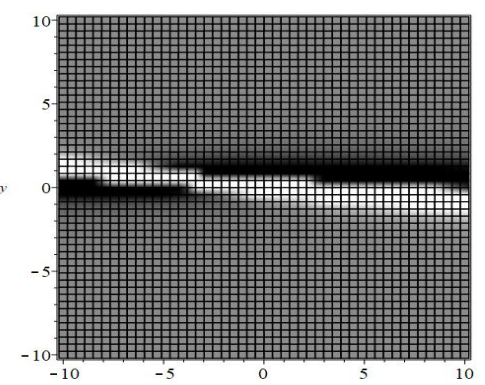

(d)

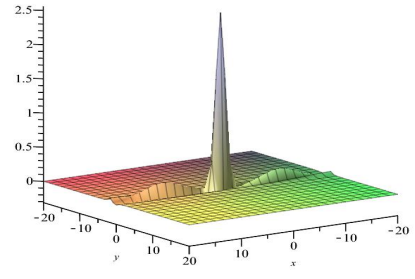

(b)

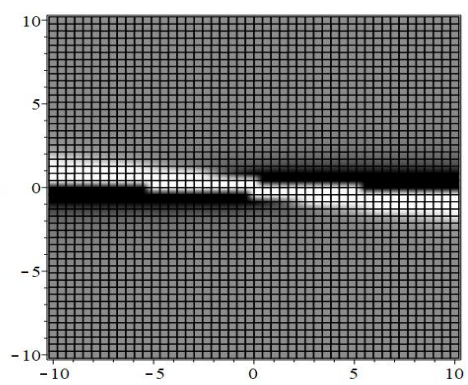

(e)

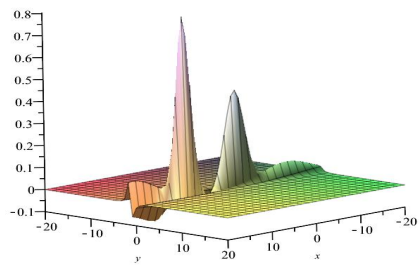

(c)

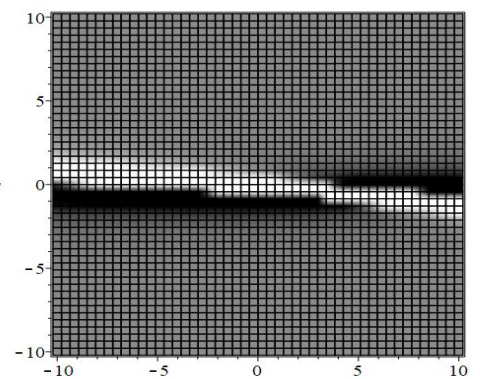

(f)

Figure 2: Three-dimensional plots and density plots of (9) with $c_{1}=-1, c_{2}=2, c_{3}=-\frac{1}{10}, a_{1}=1, a_{3}=-2, a_{5}=3, a_{6}=2$ when $t=-6$ in (a) and (d), $t=0$ in (b) and (e), $t=6$ in (c) and (f), respectively. 
Then, substituting quadratic function (11) into transformation (4), we can obtain

$$
\begin{gathered}
u_{2}=\frac{12\left(a_{1}^{2}+a_{5}^{2}\right)}{\left(a_{1} x+a_{2} y+a_{3} t+a_{4}\right)^{2}+\left(a_{5} x+a_{6} t\right)^{2}+a_{9}} \\
-\frac{24\left[\left(a_{1} x+a_{2} y+a_{3} t+a_{4}\right) a_{1}+\left(a_{5} x+a_{6} t\right) a_{5}\right]^{2}}{\left[\left(a_{1} x+a_{2} y+a_{3} t+a_{4}\right)^{2}+\left(a_{5} x+a_{6} t\right)^{2}+a_{9}\right]^{2}} .
\end{gathered}
$$

Fig. 3 gives three dimensional plots of function (12) and its density plots with a series of parameters $c_{1}=1.3, c_{2}=2.1, c_{3}=1.5, a_{3}=0.5, a_{4}=-3, a_{6}=1$ when $t=-1,0,1$.

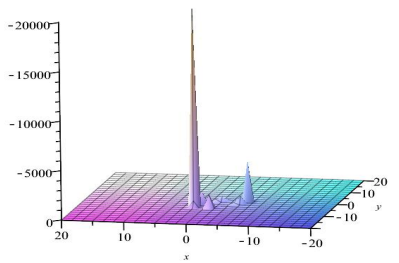

(a)

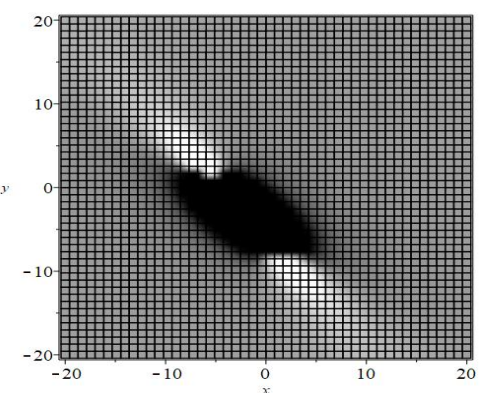

(d)

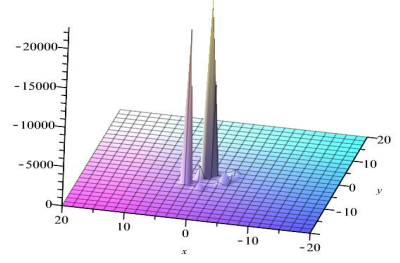

(b)

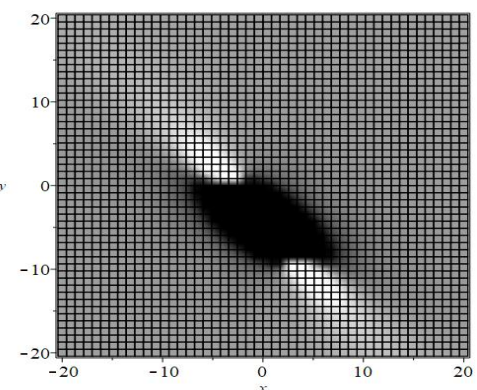

(e)

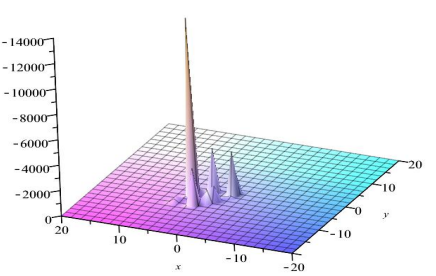

(c)

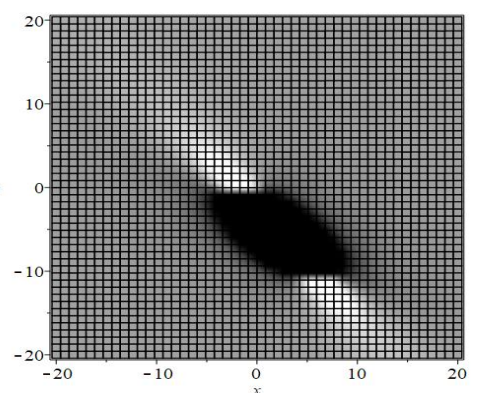

(f)

Figure 3: Three-dimensional plots and density plots of (12) with $c_{1}=1.3, c_{2}=2.1, c_{3}=1.5, a_{3}=0.5, a_{4}=-3, a_{6}=1$ when $t=-1$ in (a) and (d), $t=0$ in (b) and (e), $t=1$ in (c) and (f), respectively.

\section{Case 3.}

$$
\begin{aligned}
& a_{1}=a_{1}, a_{2}=-\frac{a_{1} a_{3} c_{2}+c_{1}\left(a_{3}^{2}-a_{8}^{2}\right)}{c_{3} a_{1}}, a_{3}=a_{3}, a_{4}=a_{4}, a_{5}=0, a_{6}=0, \\
& a_{7}=-\frac{a_{8}\left(a_{1} c_{2}+2 a_{3} c_{1}\right)}{c_{3} a_{1}}, a_{8}=a_{8}, a_{9}=-\frac{3 a_{1}^{4}}{c_{1} a_{8}^{2}}, b_{i}=0(1 \leq i \leq 4) .
\end{aligned}
$$

where $a_{1} \neq 0, a_{8} \neq 0$.

Therefore, by comparing equation (6) and limiting conditions (13), we can write a type of quadratic function solutions of equation (5)

$$
f_{3}=\left(a_{1} x+a_{2} y+a_{3} t+a_{4}\right)^{2}+\left(a_{7} y+a_{8} t\right)^{2}+a_{9} .
$$

Then, substituting quadratic function (14) into transformation (4), we can obtain

$$
u_{3}=\frac{12 a_{1}^{2}\left[\left(a_{1} x+a_{2} y+a_{3} t+a_{4}\right)^{2}+\left(a_{7} y+a_{8} t\right)^{2}+a_{9}\right]-24\left(a_{1} x+a_{2} y+a_{3} t+a_{4}\right)^{2} a_{1}^{2}}{\left[\left(a_{1} x+a_{2} y+a_{3} t+a_{4}\right)^{2}+\left(a_{7} y+a_{8} t\right)^{2}+a_{9}\right]^{2}} .
$$


Fig. 4 gives three dimensional plots of function (15) and its density plots with a series of parameters $c_{1}=1, c_{2}=2, c_{3}=1.8, a_{1}=4, a_{3}=0.6, a_{4}=-5, a_{7}=1$ when $t=-6,0,6$.

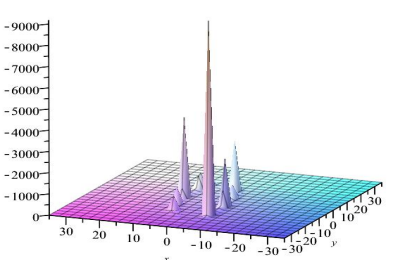

(a)

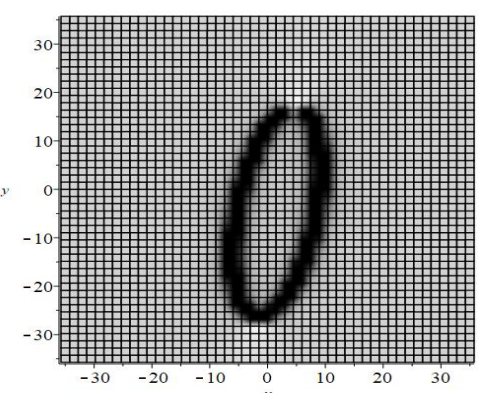

(d)

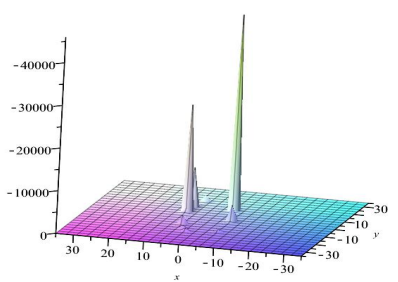

(b)

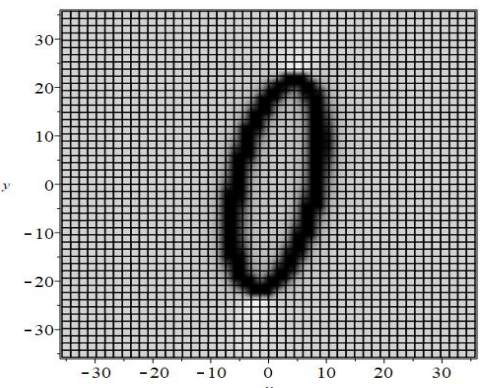

(e)

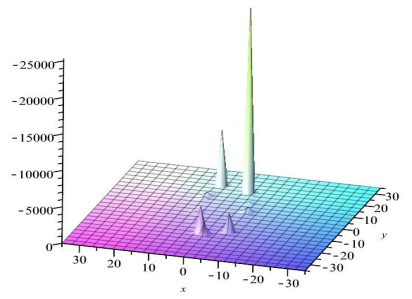

(c)

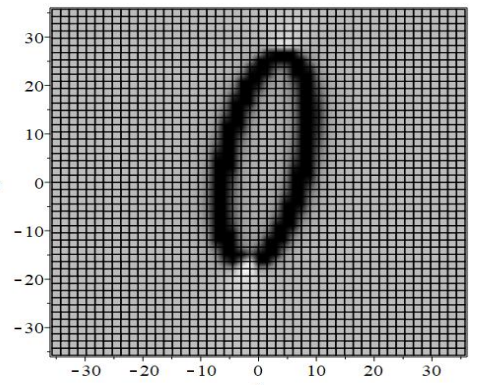

(f)

Figure 4: Three-dimensional plots and density plots of (15) with $c_{1}=1, c_{2}=2, c_{3}=1.8, a_{1}=4, a_{3}=0.6, a_{4}=-5, a_{7}=1$ when $t=-6$ in (a) and (d), $t=0$ in (b) and (e), $t=6$ in (c) and (f), respectively.

\section{Case 4.}

$$
\begin{aligned}
& a_{1}=a_{1}, a_{2}=a_{2}, a_{3}=0, a_{4}=\sqrt{\frac{3 c_{1}^{2} a_{1}^{2} a_{6}^{2}-a_{1} a_{2} a_{9} c_{2}^{2} c_{3}+a_{2}^{2} a_{9} c_{1} c_{3}^{2}}{a_{1} a_{2}^{3} c_{3}^{3}+a_{1} a_{2} a_{6}^{2} c_{2}^{2} c_{3}-a_{2}^{2} a_{6}^{2} c_{1} c_{3}^{2}} a_{6},} \\
& a_{5}=-\frac{a_{1} a_{6} c_{2}}{c_{3} a_{2}}, a_{6}=a_{6}, a_{7}=\frac{-a_{1} a_{2}^{2} c_{3}^{2}+a_{6}^{2}\left(a_{2} c_{1} c_{3}-a_{1} c_{2}^{2}\right)}{\sqrt{-a_{1}^{2}\left(a_{2}^{2} c_{3}^{2}+a_{6}^{2} c_{2}^{2}\right)+a_{1} a_{2} a_{6}^{2} c_{1} c_{3}} c_{3}}, a_{8}=0, \\
& a_{9}=a_{9}, b_{1}=0, b_{2}=0, b_{3}=\frac{\sqrt{-a_{1}^{2}\left(a_{2}^{2} c_{3}^{2}+a_{6}^{2} c_{2}^{2}\right)+a_{1} a_{2} a_{6}^{2} c_{1} c_{3}}}{c_{3} a_{2}}, b_{4}=0 .
\end{aligned}
$$

where $\sqrt{-a_{1}^{2}\left(a_{2}^{2} c_{3}^{2}+a_{6}^{2} c_{2}^{2}\right)+a_{1} a_{2} a_{6}^{2} c_{1} c_{3}}>0, a_{2} \neq 0$.

Therefore, by comparing equation (6) and limiting conditions (16), we can write a type of quadratic function solutions of equation (5)

$$
f_{4}=\left(a_{1} x+a_{2} y+a_{4}\right)^{2}+\left(a_{5} x+a_{6} t\right)^{2}+\left(b_{3} x+a_{7} y\right)^{2}+a_{9} .
$$

Then, substituting quadratic function (17) into transformation (4), we can obtain

$$
\begin{aligned}
u_{4}=\quad & \frac{12\left(a_{1}^{2}+a_{5}^{2}+b_{3}^{2}\right)}{\left(a_{1} x+a_{2} y+a_{4}\right)^{2}+\left(a_{5} x+a_{6} t\right)^{2}+\left(b_{3} x+a_{7} y\right)^{2}+a_{9}} \\
& -\frac{24\left[\left(a_{1} x+a_{2} y+a_{4}\right) a_{1}+\left(a_{5} x+a_{6} t\right) a_{5}+\left(b_{3} x+a_{7} y\right) b_{3}\right]^{2}}{\left[\left(a_{1} x+a_{2} y+a_{4}\right)^{2}+\left(a_{5} x+a_{6} t\right)^{2}+\left(b_{3} x+a_{7} y\right)^{2}+a_{9}\right]^{2}} .
\end{aligned}
$$




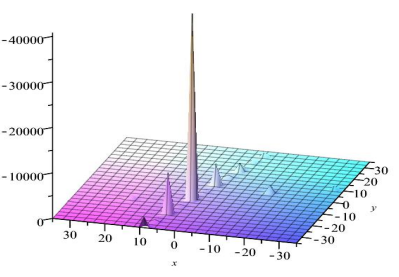

(a)

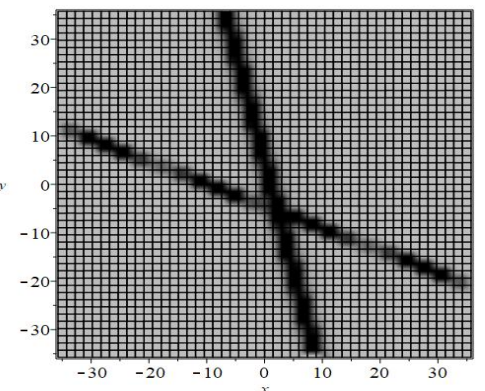

(d)

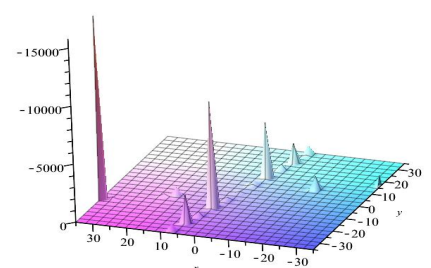

(b)

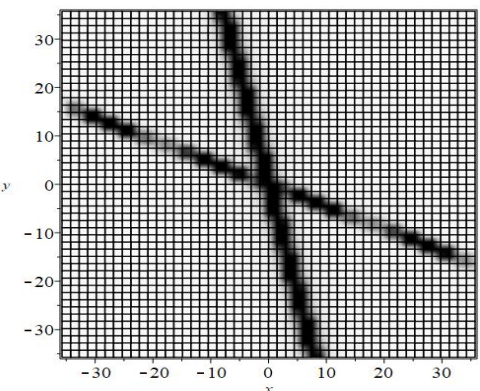

(e)

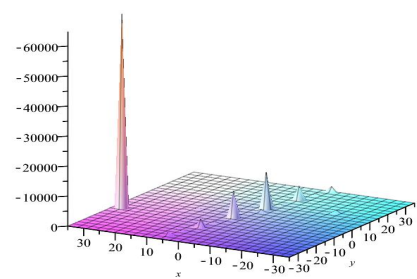

(c)

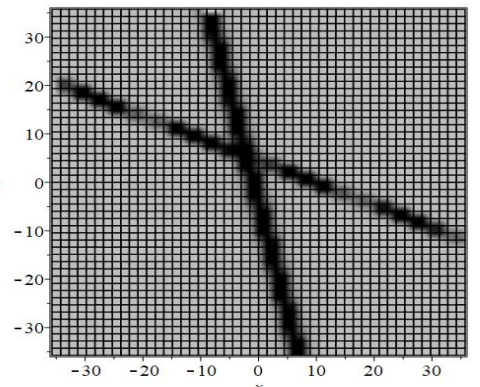

(f)

Figure 5: Three-dimensional plots and density plots of (18) with $c_{1}=-5, c_{2}=-2, c_{3}=1, a_{1}=1.5, a_{2}=0.6, a_{6}=-5, a_{9}=1$ when $t=-11$ in (a) and (d), $t=0$ in (b) and (e), $t=11$ in (c) and (f), respectively.

Fig. 5 gives three dimensional plots of function (18) and its density plots with a series of parameters $c_{1}=-5, c_{2}=-2, c_{3}=1, a_{1}=1.5, a_{2}=0.6, a_{6}=-5, a_{9}=1$ when $t=-11,0,11$.

\section{Multi lump solutions of equation}

Now for equation (1), one can consder its multi lump solutions and function $f$ is set to [8]

$$
f(x, y, t)=e^{-\xi_{1}}+a_{1} e^{\xi_{1}}+a_{2} \sin \left(\xi_{2}\right) .
$$

with $\xi_{1}=k_{1}\left(x+r_{1} y-w_{1} t\right), \xi_{2}=k_{2}\left(r_{2} y-w_{2} t\right)$.

Substituting (19) into bilinear form (5), we are able to get algebraic systems for $a_{1}, a_{2}, k_{1}, r_{1}, w_{1}, k_{2}, r_{2}, w_{2}$, and the relationship is as below:

$$
\begin{aligned}
& a_{1}=\frac{k_{2}^{2} a_{2}^{2}\left[-3\left(k_{1}^{2}+k_{2}^{2}\right)^{2}+c_{1}\left(w_{1}-w_{2}\right)^{2} k_{1}^{2}\right]}{4 k_{1}^{2}\left[3\left(k_{1}^{2}+k_{2}^{2}\right)^{2}+c_{1}\left(w_{1}-w_{2}\right)^{2} k_{2}^{2}\right]}, a_{2}=a_{2}, k_{1}=k_{1}, k_{2}=k_{2}, w_{1}=w_{1}, \\
& w_{2}=w_{2}, r_{1}=\frac{3 k_{2}^{4}+\left[2 k_{1}^{2}+\left(-2 c_{1} w_{2}+c_{2}\right) w_{1}+c_{1} w_{2}^{2}\right] k_{2}^{2}-k_{1}^{4}+\left(-c_{1} w_{1}^{2}+c_{2} w_{1}\right) k_{1}^{2}}{c_{3}\left(k_{1}^{2}+k_{2}^{2}\right)} \\
& r_{2}=\frac{-3 k_{1}^{4}+\left[-2 k_{2}^{2}+\left(-2 c_{1} w_{1}+c_{2}\right) w_{2}+c_{1} w_{1}^{2}\right] k_{1}^{2}+k_{2}^{4}+\left(-c_{1} w_{2}^{2}+c_{2} w_{2}\right) k_{2}^{2}}{c_{3}\left(k_{1}^{2}+k_{2}^{2}\right)}
\end{aligned}
$$

where $k_{1} k_{2} \neq 0, w_{1} w_{2} \neq 0$.

Therefore, by comparing equation (19) and limiting conditions (20), we can write a type of multi lump solutions of equation (5)

$$
f_{5}=e^{-\left[k_{1}\left(x+r_{1} y-w_{1} t\right)\right]}+a_{1} e^{k_{1}\left(x+r_{1} y-w_{1} t\right)}+a_{2} \sin \left[k_{2}\left(x+r_{2} y-w_{2} t\right)\right] .
$$


Then, substituting exponential trigonometric function (21) into transformation (4), we can obtain

$$
u_{5}=\frac{6\left[k_{1}^{2} e^{-\xi_{1}}+a_{1} k_{1}^{2} e^{\xi_{1}}-a_{2} k_{2}^{2} \sin \left(\xi_{2}\right)\right]}{e^{-\xi_{1}}+a_{1} e^{\xi_{1}}+a_{2} \sin \left(\xi_{2}\right)}-\frac{6\left[-k_{1} e^{-\xi_{1}}+a_{1} k_{1} e^{\xi_{1}}+a_{2} k_{2} \cos \left(\xi_{2}\right)\right]^{2}}{\left[e^{-\xi_{1}}+a_{1} e^{\xi_{1}}+a_{2} \sin \left(\xi_{2}\right)\right]^{2}}
$$

Fig. 6 gives the plots of $(22)$ and its density plots for the parameters $c_{1}=-15.75, c_{2}=$ $-660.5, c_{3}=1386, k_{1}=1, k_{2}=-1, a_{2}=2, w_{1}=-2, w_{2}=2$ when $t=-2,0,2$.

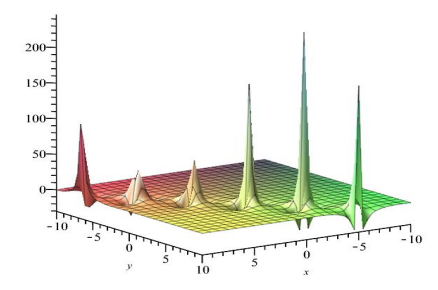

(a)

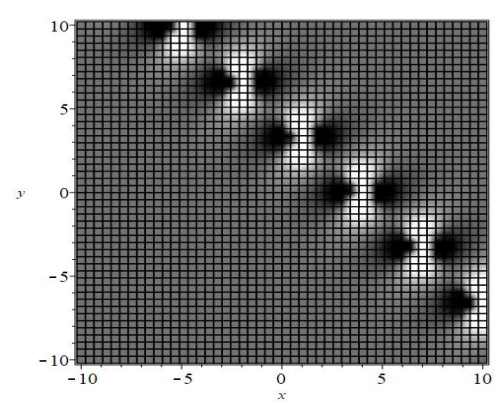

(d)

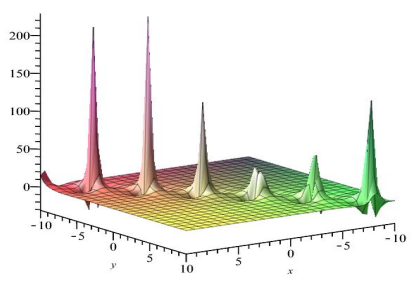

(b)

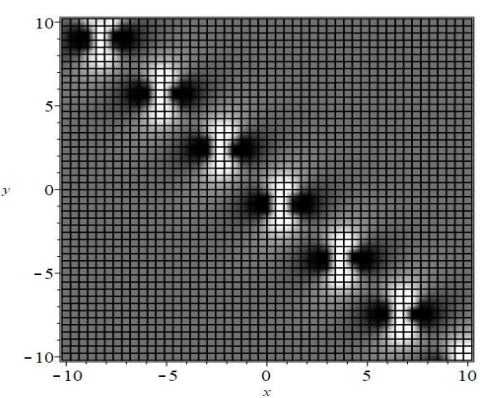

(e)

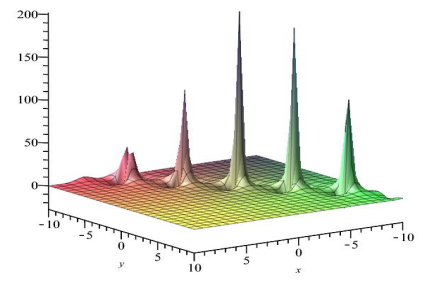

(c)

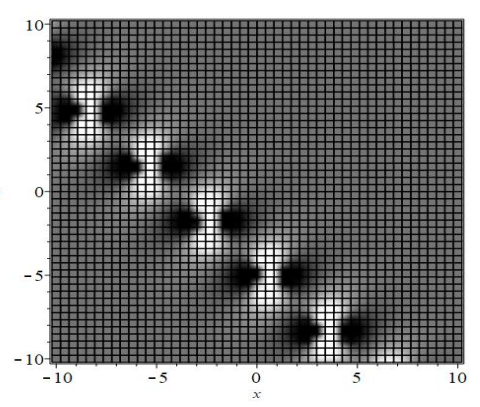

(f)

Figure 6: Three-dimensional plots and density plots of (18) with parameters $c_{1}=-15.75, c_{2}=-660.5, c_{3}=1386, k_{1}=1, k_{2}=-1, a_{2}=$ $2, w_{1}=-2, w_{2}=2$, when $t=-2$ in (a) and (d), $t=0$ in (b) and (e), $t=2$ in (c) and (f), respectively.

\section{More soliton solution}

In order to find some new soliton solutions for equation(1), we will set the function $u$ in the form of trigonometric function and hyperbolic function [3].

\section{1 the improved tanh-coth method}

Through calculating equation (1), one can find the equality for solving the value of $\mathrm{n}$ is $n+4=$ $n+n+2$, so $n=2$.

Taking

$$
u=a_{0}+a_{1} \tanh (\xi)+a_{2}[\tanh (\xi)]^{2}+a_{3}[\tanh (\xi)]^{-3}+a_{4}[\tanh (\xi)]^{-2},
$$

with $\xi=k x+r y-w t$. Substitute trigonometric hyperbolic function (23) into equation (1) and collect all coefficients of $(\tanh (\xi))^{j}$, where $j=-1,-2,-3,-4,-5,-6,-7,-8$ and $0,1,2,3,4,5,6$. Then making these coefficients are equal to zero, and creating an algebraic 
system about $a_{0}, a_{1}, a_{2}, a_{3}, a_{4}, w$. Finally, we can get

$$
\begin{aligned}
& k=k, r=r, w=w, a_{0}=\frac{8 k^{4}-k r c_{3}+k w c_{2}-c_{1} w^{2}}{2 c_{4} k^{2}}, \\
& a_{1}=0, a_{2}=-\frac{6 k^{2}}{c_{4}}, a_{3}=0, a_{4}=-\frac{6 k^{2}}{c_{4}} .
\end{aligned}
$$

Therefore, we can derive the solution

$$
u_{6}=a_{0}+a_{2}[\tanh (k x+r y-w t)]^{2}+a_{4}[\tanh (k x+r y-w t)]^{-2} .
$$

Fig. 7 gives the plots of (25) and its density plots for the parameters $c_{1}=-4, c_{2}=2, c_{3}=$ $-1, c_{4}=6, k=0.1, r=0.2, w=-1$ when $t=-4,0,4$.

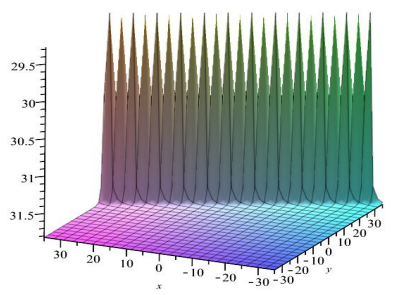

(a)

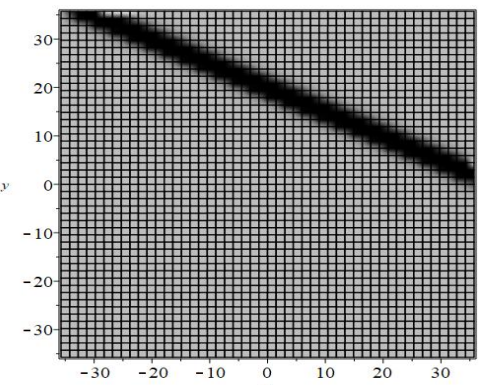

(d)

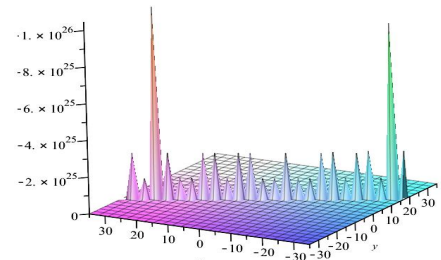

(b)

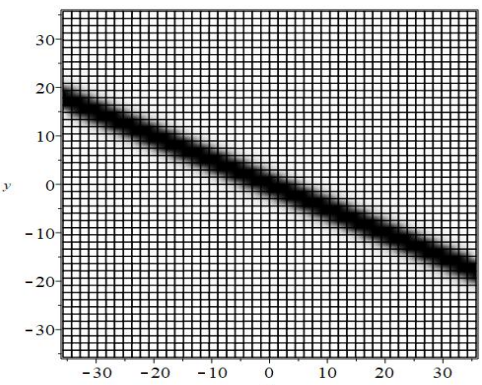

(e)

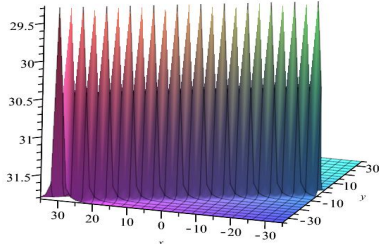

(c)

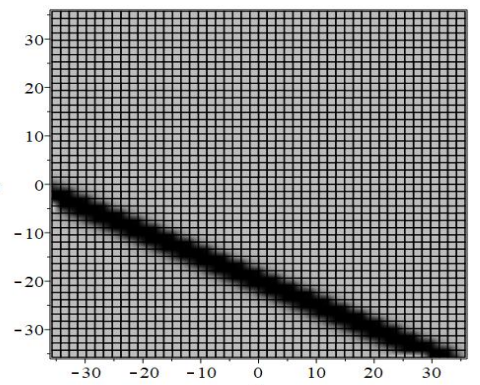

(f)

Figure 7: Three-dimensional plots and density plots of (25) with $c_{1}=-4, c_{2}=2, c_{3}=-1, c_{4}=6, k=0.1, r=0.2, w=-1$ while $t=-4$ in (a) and (d), $t=0$ in (b) and (e), $t=4$ in (c) and (f), respectively.

\section{2 the improved tah-cot method}

In a like manner, we can assume the function $\mathrm{u}$ in the form

$$
u=a_{0}+a_{1} \tan (\xi)+a_{2}[\tan (\xi)]^{2}+a_{3}[\tan (\xi)]^{-3}+a_{4}[\tan (\xi)]^{-2},
$$

where $\xi=k x+r y-w t$.

Substitute trigonometric hyperbolic function (26) into equation (1) and collect all coefficients of $(\tan (\xi))^{j}$, where $j=-1,-2,-3,-4,-5,-6,-7,-8$ and $0,1,2,3,4,5,6$. Then making these coefficients are equal to zero, and creating an algebraic system about $a_{0}, a_{1}, a_{2}, a_{3}, a_{4}, w$. Finally, we can get

$$
k=k, r=-\frac{8 k^{4}+2 c_{4} k^{2} a_{0}-k w c_{2}+c_{1} w^{2}}{k c_{3}}, w=w
$$




$$
a_{0}=a_{0}, a_{1}=0, a_{2}=-\frac{6 k^{2}}{c_{4}}, a_{3}=0, a_{4}=-\frac{6 k^{2}}{c_{4}} .
$$

Therefore, we can derive the solution

$$
u_{7}=a_{0}+a_{2}[\tan (k x+r y-w t)]^{2}+a_{4}[\tan (k x+r y-w t)]^{-2} .
$$

Fig. 8 gives the plots of (28) and its density plots for the parameters $c_{1}=-4, c_{2}=2, c_{3}=$ $-1.08, c_{4}=6, k=0.1, w=-1, a_{0}=31.84$ when $t=-4,0,4$.

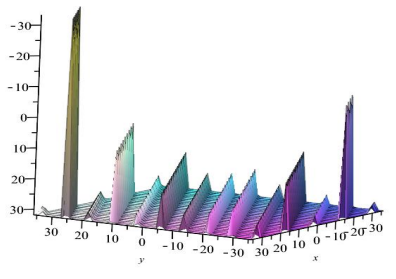

(a)

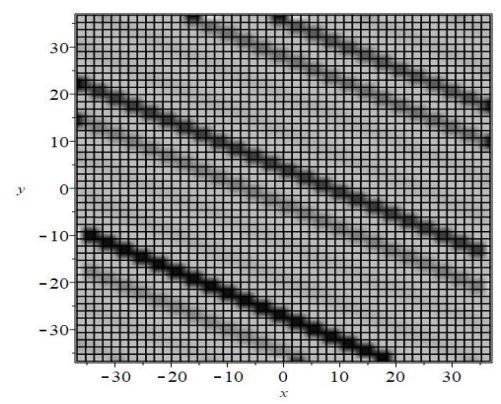

(d)

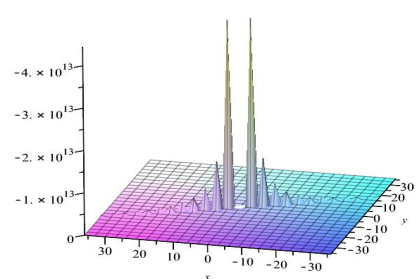

(b)

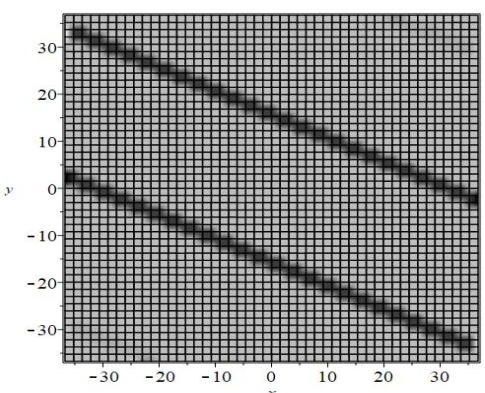

(e)

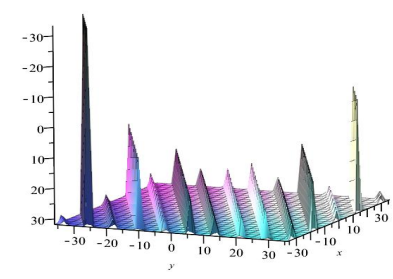

(c)

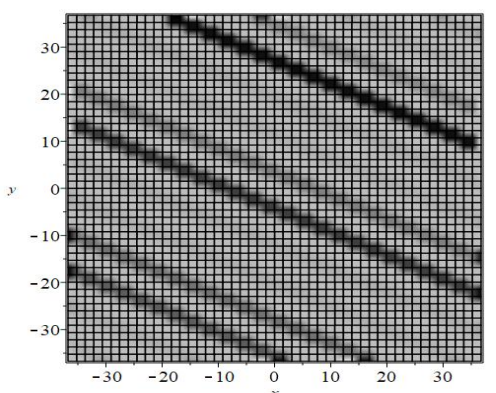

(f)

Figure 8: Three-dimensional plots and density plots of (28) with parameters $c_{1}=-4, c_{2}=2, c_{3}=-1.08, c_{4}=6, k=0.1, w=-1, a_{0}=$ 31.84 while $t=-4$ in (a) and (d), $t=0$ in (b) and (e), $t=4$ in (c) and (f), respectively.

\section{Conclusion}

To sum up, we discuss the $(2+1)$ nonlinear evolution equation by making use of bilinear method, test function, improved tan-cot and tanh-coth method. At the same time, through using the powerful computing power of software Maple, we give lump solutions, multi lump solutions and more soliton solutions with non-zero real number $c_{1}, c_{2}, c_{3}, c_{4}$. By determining different parameters, the three-dimensional diagram and density diagram of each solution obtained in this paper are drawn in Figure 1-8, which is proved that this technology is effective in dealing with nonlinear evolution equations.

\section{Acknowledgements}

The work is supported by the National Natural Science Foundation of China (project No. 11371086, 11671258, 11975145), the Fund of Science and Technology Commission of Shanghai 
Municipality (project No. 13ZR1400100), the Fund of Donghua University, institute for nonlinear sciences and the Fundamental Research Funds for the Central Universities with contract number $2232021 \mathrm{G}-13$.

\section{References}

[1] Ablowitz, M. J., Clarkson, P. A.: Solitons, Nonlinear Evolution Equations and Inverse Scattering. Cambridge University Press, New York, 1991.

[2] Ali, K. K., Mehanna, M. S.: Traveling wave solutions and numerical solutions of GilsonPickering equation. Results in Physics, 28:104596, (2021).

[3] Ali, M. R., Sadat, R.: Construction of Lump and optical solitons solutions for $(3+1)$ model for the propagation of nonlinear dispersive waves in inhomogeneous media. Optical and Quantum Electronics, 53(6):279, (2021).

[4] Chen, Shou-Ting, Ma, Wen-Xiu: Lump solutions to a generalized BogoyavlenskyKonopelchenko equation. Frontiers of Mathematics in China, 13(3):525-534, (2018).

[5] Dai, Zhengde, Liu, Jun, Liu, Zhenjiang: Exact periodic kink-wave and degenerative soliton solutions for potential Kadomtsev-Petviashvili equation. Communications in Nonlinear Science and Numerical Simulation, 15(9):2331-2336, (2010).

[6] Gilson, C.R, Nimmo, J.J.C.: Lump solutions of the BKP equation. Physics Letters A, 147(8-9):472-476, (1990).

[7] Hirota, R.: The direct method in soliton theory. Cambridge University Press, Cambridge, United Kingdom, (2004).

[8] Hossen, M. Belal, Roshid, Harun-Or, Ali, M. Zulfikar: Multi-soliton, breathers, lumps and interaction solution to the $(2+1)$-dimensional asymmetric Nizhnik-Novikov-Veselov equation. Heliyon, 5(10):e02548, (2019).

[9] Ma, Hong-Cai, Bai, Yun-Xiang, Deng, Ai-Ping: Multiple lump solutions of the (2+1)-dimensional Konopelchenko-Dubrovsky equation. Mathematical Methods in the Applied Sciences, 43(12):7135-7142, (2020).

[10] Ma, Hong-Cai, Bai, Yun-Xiang, Deng, Ai-Ping: Multiple Lump Solutions of the (4+1)Dimensional Fokas Equation. Advances in Mathematical Physics, 2020:3407676, (2020).

[11] Ma, Hong-Cai, Cheng, Qiao-Xin Deng, Ai-Ping: Solitons, Breathers, and Lump Solutions to the (2+1)-Dimensional Generalized Calogero-Bogoyavlenskii-Schiff Equation. Complexity, 2021:7264345, (2021).

[12] Ma, Hong-Cai, Deng, Ai-Ping: Lump Solution of (2+1)-Dimensional Boussinesq Equation. Communications in Theoretical Physics, 65(5):546-552, (2016).

[13] Ma, Hong-Cai, Wu, Han-Fang, Ma, Wen-Xiu, Deng, Ai-Ping: Localized interaction solutions of the (2+1)-dimensional Ito Equation. Optical and Quantum Electronics, 53(6):303, (2021). 
[14] Ma, Hong-Cai, Wu, Han-Fang, Ma, Wen-Xiu, Deng, Ai-Ping: Lump and Interaction Solutions of the (2+1)-dimensional bSK equation. East Asian Journal on Applied Mathematics, (June):180121, (2021).

[15] Ma, Wen-Xiu: Lump solutions to the Kadomtsev-Petviashvili equation. Physics Letters A, 379(36):1975-1978, (2015).

[16] Ma, Wen-Xiu, Qin, Zhenyun, Lü Xing: Lump solutions to dimensionally reduced p-gKP and p-gBKP equations. Nonlinear Dynamics, 84(2):923-931, (2016).

[17] Ma, Wen-Xiu, Zhou, Yuan, Dougherty, Rachael: Lump-type solutions to nonlinear differential equations derived from generalized bilinear equations. International Journal of Modern Physics B, 30:1640018, (2016).

[18] Manakov, S. V., Zakharov V. E., Bordag, L. A., Its, A. R., Matveev, V. B.: Two-dimensional solitons of the Kadomtsev-Petviashvili equation and their interaction. Physics Letters A, 63(3):205-206, (1977).

[19] Peng, Wei-Qi, Tian, Shou-Fu, Zhang, Tian-Tian: Analysis on lump, lumpoff and rogue waves with predictability to the $(2+1)$-dimensional B-type Kadomtsev-Petviashvili equation. Physics Letters A, 382(38):2701-2708, (2018).

[20] Rao, Ji Guang, Liu, Yao Bin, Qian, Chao, He, Jing Song: Rogue waves and hybrid solutions of the boussinesq equation. Zeitschrift fur Naturforschung - Section A Journal of Physical Sciences, 72(4):307-314, (2017).

[21] Tan, Wei, Dai, Zhengde: Spatiotemporal dynamics of lump solution to the (1+1)dimensional Benjamin-Ono equation. Nonlinear Dynamics, 89(4):2723-2728, (2017).

[22] Wang, Chuanjian: Dynamic behavior of traveling waves for the Sharma-Tasso-Olver equation. Nonlinear Dynamics, 85(2):1119-1126, (2016).

[23] Wang, Chuanjian: Lump solution and integrability for the associated Hirota bilinear equation. Nonlinear Dynamics, 87(4):2635-2642, (2017).

[24] Wang, Hui: Lump and interaction solutions to the (2+1)-dimensional Burgers equation. Applied Mathematics Letters, 85:27-34, (2018).

[25] Yue, Yunfei, Huang, Lili, Chen, Yong: N-solitons, breathers, lumps and rogue wave solutions to a $(3+1)$-dimensional nonlinear evolution equation. Computers and Mathematics with Applications, 75(7):2538-2548, (2018). 